\title{
L'uso di body shaming è una vergogna?
}

\author{
Edoardo Lombardi Vallauri
}

PUBBLICATO: 31 MAGGIO 2019

\section{Quesito:}

Il signor Gabriele P., di Napoli, chiede se esiste un'espressione italiana per tradurre l'inglese body shaming.

\section{L'uso di body shaming è una vergogna?}

I nsieme al suo diffondersi sul web, l'uso poco gentile di criticare le persone per aspetti del loro corpo ha cominciato a essere menzionato anche da noi italiani mediante l'espressione inglese body shaming, forma sostantivata del verbo to shame, identico a shame 'vergogna', che sfrutta la straordinaria elasticità di questa lingua nel consentire allo stesso termine di figurare virtualmente in qualsiasi classe di parole. Questa possibilità è spesso maggiore nelle lingue dette "a morfologia isolante", cioè quelle in cui le parole si modificano pochissimo o per niente, come l'inglese e, ancor più, il cinese mandarino o il vietnamita. Ad esempio, l'inglese presenta in tutto il paradigma di un verbo regolare una manciata di forme (connect - connects - connected - connecting), neanche paragonabile alla complessità morfologica esibita da altre lingue, compreso l'italiano. In compenso, l'inglese può "piegare" le sue parole a svolgere molte più funzioni.

Questo fenomeno si chiama conversione, ed è in virtù di esso che in inglese shame non è solo il sostantivo che significa 'vergogna', ma anche il verbo (to shame) che significa 'generare vergogna', quindi 'far vergognare', 'umiliare'. Alcune parole in inglese possono fare molto di più. Ad esempio round è aggettivo: a round face 'un viso rotondo'; nome: a round 'un cerchio, un giro' (a round of drinks, un giro di bevute); preposizione: round a table 'intorno a un tavolo', round the corner 'dietro l'angolo' (cioè, girato l'angolo); avverbio: to walk round and round 'camminare in tondo'; e verbo: to round Cape Horn 'doppiare (aggirare) il capo Horn'.

Tornando alla nostra questione, l'italiano da vergogna deriva il verbo svergognare, il cui senso però include abbastanza necessariamente l'idea dello smascheramento, cioè che la vergogna gettata su qualcuno sia giustificata: lo ha svergognato fa pensare che sia stato rivelato qualcosa di cui chiunque si dovrebbe vergognare. Invece il verbo inglese significa semplicemente 'creare vergogna', 'far vergognare', anche nei casi in cui questa vergogna non sia giustificata, e la persona che si vergogna finisca per sentirsi così non tanto perché ci sia in lei qualcosa di sbagliato, ma semplicemente perché è stata esposta a una critica in pubblico. Ė appunto il caso del body shaming sul web, dove l'oggetto della critica può essere una corporatura non ideale, un tatuaggio, o qualsiasi altra caratteristica del corpo che non giustifica di per sé la vergogna, ma può condurvi per il modo o la circostanza in cui viene commentata.

Non è una buona traduzione neanche umiliare il corpo (di un altro), che talora viene proposta sul web, perché questa espressione evoca un'azione fisica di sottomissione del corpo altrui o di violenza su di esso, piuttosto che una critica verbale rivolta a sue caratteristiche.

Invece, grazie anche alla capacità dell'inglese di giustapporre due parole creando un breve composto equivalente a quello che l'italiano ottiene aggiungendovi almeno una preposizione e spesso l'articolo, 
body shaming, forma sostantivata del verbo to shame, significa assai in breve 'il far vergognare del (proprio) corpo'.

Chi non voglia dunque adoperare l'espressione inglese, scartati svergognare (per) il corpo e umiliare il corpo con le ragioni di significato che abbiamo dette, dovrà ricorrere a perifrasi un po' più lunghe, come far vergognare una persona del suo corpo o umiliazione di qualcuno riguardo al suo corpo; oppure meglio, spostando il centro semantico dal destinatario dell'azione a chi la compie, potrà dire derisione del corpo (altrui), perché nella semantica del verbo deridere è presente l'idea che chi subisce quell'azione provi un disagio.

Tutto sommato, viene da formulare l'auspicio che l'esigenza di esprimere questo significato nella comunità che parla italiano resti marginale, e che quindi ci si possa accontentare di un uso occasionale del termine inglese, seguitando a marcarne l'estraneità alla nostra cultura.

\section{Cita come:}

Edoardo Lombardi Vallauri, L'uso dibody shaming è una vergogna?, "Italiano digitale", 2019, IX, 2019/2 (aprile-giugno)

DOI: $10.35948 / 2532-9006 / 2020.3120$

Copyright 2019 Accademia della Crusca

Pubblicato con licenza creative commons CC BY-NC-ND 